\title{
Positive Stable Frailty Approach in the Construction of Dependence Life-Tables
}

\author{
Onchere Walter ${ }^{1 *}$ (), Weke Patrick ${ }^{2}$, Joseph Ottieno², Ogutu Carolyne ${ }^{2}$ \\ ${ }^{1}$ Department of Mathematics and Actuarial Science, Kisii University, Kisii, Kenya \\ ${ }^{2}$ School of Mathematics, University of Nairobi, Nairobi, Kenya \\ Email: ^walonc2006@kisiiuniversity.ac.ke
}

How to cite this paper: Walter, O., $\mathrm{Pa}$ trick, W., Otieno, J. and Carolyne, O. (2021) Positive Stable Frailty Approach in the Construction of Dependence Life-Tables. Open Journal of Statistics, 11, 506-523. https://doi.org/10.4236/ojs.2021.114032

Received: March 15, 2021

Accepted: August 13, 2021

Published: August 16, 2021

Copyright $\odot 2021$ by author(s) and Scientific Research Publishing Inc. This work is licensed under the Creative Commons Attribution International License (CC BY 4.0).

http://creativecommons.org/licenses/by/4.0/ (c) (i) Open Access

\begin{abstract}
Dependence may arise in insurance when the insureds are clustered into groups e.g. joint-life annuities. This dependence may be produced by sharing a common risk acting on mortality of members of the group. Various dependence models have been considered in literature; however, the focus has been on either the lower-tail dependence alone or upper-tail dependence alone. This article implements the frailty dependence approach to life insurance problems where most applications have been within medical setting. Our strategy is to use the conditional independence assumption given an observed association measure in a positive stable frailty approach to account for both lower and upper-tail dependence. The model is calibrated on the association of Kenyan insurers 2010 male and female published rates. The positive stable model is then proposed to construct dependence life-tables and generate life annuity payment streams in the competitive Kenyan market.
\end{abstract}

\section{Keywords}

Joint-Life Annuity, Life-Table Functions, Shared Frailty Model, Positive Stable Distribution, Bayesian Inference

\section{Introduction and Motivation}

When annuity payments are concerned, the calculation of expected present values (EPVs), needed in pricing and reserving, requires an appropriate mortality model in order to avoid biased valuations [1] [2] [3]. Frailty models are used in life insurance to represent heterogeneity in a population due to unreported risk factors [4] [5]. Heterogeneity due to reported risk factors is addressed at policy issue during the underwriting process. There has been a growing literature on dependence mortality modeling in life insurance in recent years (see, e.g., [6] [7] 
[8]) where the focus has been on either the lower-tail dependence alone or upper-tail dependence alone. Frailty dependence modeling [9] [10] [11] [12] is an approach that accounts for dependence in event times of related individuals. Clustered survival times are assumed to be conditional independent with respect to the shared risk. Our strategy is to use the conditional independence assumption to account for lower and upper-tail dependence for a given association measure. Dependence frailty models have been used by several authors with applications in medical field (see e.g., [13]). This article implements the dependence frailty approach in insurance risk management setting.

Our article contributes to the existing literature in several ways. First, we apply a shared frailty approach to life insurance risk problems where most applications have been within medical setting. Second, most dependence models in literature have focused on either the lower-tail dependence alone or upper-tail dependence alone. We apply the conditional independence assumption for an observed association measure in a positive stable frailty to account for both lower and upper-tail dependence. Third, we incorporate a stochastic dependence structure via a dynamically evolving positive stable process to model time-varying shared risk.

\section{Notations, Assumptions and Data}

\subsection{Notations}

To facilitate an easier discussion of frailty dependence modeling, the following notations are used. We consider joint-life annuity contracts where $x$ is the age of the male annuitant whose future lifetime random variable is $t_{i 1}$ and $y$ the age of the female annuitant whose future lifetime random variable is $t_{i 1} \cdot v^{t}$ is the present value factor, $s_{\overline{x y}}(t)$ is the probability of survival until the last of $(x, y)$ dies and $a_{\bar{x} y}$ the EPV of the benefit. $D_{x y}=v^{x y} l_{x y}$ and $N_{x y}=\sum_{k=0}^{\infty} D_{x y+k}$ are commutation functions.

\subsection{Assumptions}

For simplicity of notation of the commutation functions, we suppose that the limiting age of our mortality table is infinity.

The force of mortality $\lambda_{x}(t)$ for a life aged $x$ during time $t$ is assumed piece-wise constant across each whole year of age $[t, t+1)$. i.e. $\lambda_{x}(t)=\lambda_{x+z}(t) ; 0<z<1$. Similar assumptions are found in [14].

A deterministic financial structure is adopted for the present value factor $v^{t}$ for illustration purposes.

Model calibration with reference to standard mortality tables [5] [15] [16] is applied due to limited joint-life mortality data-set that is available in the Kenyan annuity market.

\subsection{Data}

The association of Kenyan insurers (AKI) 2010 published mortality tables is 
based upon data collected by the association of Kenya insurers for an investigation into the mortality of assured lives in the Republic of Kenya. The AKI 2010 mortality rates are used as the baseline hazard rates in the study. Joint-life and last survivor annuitants member data for policies in-force between 2001-2013 will be used in the study to determine the average age difference for insured couples in Kenya. The annuitants data used in this article was obtained from a major Kenyan insurance company. To preserve confidentiality, we took a sub-sample of 178 joint-life policies.

\section{Materials and Methods}

\subsection{Joint-Life Last Survivor Annuity}

The proposed model can be applied to any type of joint-life annuity business. In this article, we discuss the case of joint-life last survivor annuities. This is a contract that provides level payments to two or more annuitants until the last of them dies. This can be an immediate annuity for a single lump-sum payment whose expected present value (EPV) of say amount $b$ per annum, payable in arrears, until the last of $(x, y)$ dies is given by:

$$
b a_{\overline{x y}}=b \sum_{t=1}^{\infty} v^{t} s_{\overline{x y}}(t)
$$

where the future lifetime random variable $T=\operatorname{Max}\left(t_{i 1}, t_{i 2}\right)$.

Under the dependence (frailty) assumption:

$$
s_{\overline{x y}}(t)=s_{x}(t)+s_{y}(t)-s_{x y}(t)
$$

Assuming independence we have:

$$
s_{\overline{x y}}(t)=s_{x}(t)+s_{y}(t)-s_{x}(t) \cdot s_{y}(t)
$$

The EPV in Equation (1) becomes:

$$
\begin{gathered}
b \sum_{t=1}^{\infty} v^{t}\left[\exp \left\{-\int_{0}^{t} \lambda_{x+s} \mathrm{~d} s\right\}+\exp \left\{-\int_{0}^{t} \lambda_{y+s} \mathrm{~d} s\right\}-\exp \left\{-\int_{0}^{t}\left(\lambda_{x y+s}\right) \mathrm{d} s\right\}\right] \\
b \sum_{t=1}^{\infty} v^{t}\left[\exp \left\{-\int_{0}^{t} \lambda_{x+s} \mathrm{~d} s\right\}+\exp \left\{-\int_{0}^{t} \lambda_{y+s} \mathrm{~d} s\right\}-\exp \left\{-\int_{0}^{t}\left(\lambda_{x+s}+\lambda_{y+s}\right) \mathrm{d} s\right\}\right]
\end{gathered}
$$

respectively. Here again for simplicity of notation, we suppose that the limiting age of our mortality table is infinity. If the purchase price for the annuity is $p$ then the future level payment stream $b$ applying the traditional equivalence principle is:

$$
b=\frac{p}{a_{\bar{x} y}}
$$

The prevailing traditional insurance practice assumes independence in pricing joint-life annuity contracts thereby adopting the EPV shown in Equation (5) whose joint mortality is obtained by summing up the individual mortality rates. Frailty dependence models focus on modeling the EPV as shown in Equation (4) whose joint mortality accounts for heterogeneity and dependence. 


\subsection{Shared Frailty Model}

Definition 1. The hazard function for a shared frailty model is given by,

$$
\lambda_{i j}\left(t \mid \Omega_{i}\right)=\Omega_{i} \lambda_{0}(t) ; t>0
$$

where $\lambda_{i j}\left(t \mid \Omega_{i}\right)$ is the conditional hazard function for the $f^{\text {th }}$ individual in the $i^{\text {th }}$ group and $\Omega_{i}$ is the shared random effect associated with the $i^{\text {th }}$ group. $\lambda_{0}(t)$ is the population's base force of mortality.

Formally, the expression of the bivariate net survival function is summarized in the following proposition.

Proposition 1. Under the assumption of independent future life-times for a given shared frailty the bivariate net survival function is,

$$
s\left(t_{i 1}, t_{i 2}\right)=L_{\Omega_{i}}\left(\Lambda_{01}\left(t_{i 1}\right)+\Lambda_{02}\left(t_{i 2}\right)\right)
$$

Proof. The bivariate conditional survival function for a given shared frailty $\Omega_{i}$ at time $t_{i 1}>0, t_{i 2}>0$ is given by; $s\left(t_{i 1}, t_{i 2} \mid \Omega_{i}\right)=s\left(t_{i 1} \mid \Omega_{i}\right) s\left(t_{i 2} \mid \Omega_{i}\right)$ and from $s(t \mid \Omega)=\exp \left\{-\int_{0}^{t} \lambda(u \mid \Omega) \mathrm{d} u\right\}=\exp \left(-\Omega \Lambda_{0}(t)\right)$ we have that $s\left(t_{i 1}, t_{i 2} \mid \Omega_{i}\right)=\exp \left\{-\Omega\left[\Lambda_{01}\left(t_{i 1}\right)+\Lambda_{02}\left(t_{i 2}\right)\right]\right\}$ using expectation $s\left(t_{i 1}, t_{i 2}\right)=E\left[\exp \left\{-\Omega\left[\Lambda_{01}\left(t_{i 1}\right)+\Lambda_{02}\left(t_{i 2}\right)\right]\right\}\right]$ this simplifies to $s\left(t_{i 1}, t_{i 2}\right)=L_{\Omega_{i}}\left(\Lambda_{01}\left(t_{i 1}\right)+\Lambda_{02}\left(t_{i 2}\right)\right)$

\subsection{Shared Frailty and Archimedean Copula Approach}

Copulas have been studied in actuarial science to model joint-life survival functions [17]. Similarity between the frailty and copula dependence approach is discussed with reference to the elliptical or Archimedean dependence copula. The family of Archimedean copula [18] [19] in the bivariate case is described by reference to a generator function.

$$
C_{\phi}(u, w)=p\{q(u)+q(w)\}
$$

where the generator function $p($.$) is any non-negative decreasing function and$ non-negative second derivative with $p(0)=1$ and $q($.$) its pseudo-inverse$ function. A special case showing similarity to shared frailty approach is when $p(s)=L_{\Omega}(s)$ where $\Omega$ is the frailty random variable and $u=s\left(t_{i 1}\right), w=s\left(t_{i 2}\right)$ this leads to:

$$
C_{\phi}\left\{s\left(t_{i 1}\right), s\left(t_{i 2}\right)\right\}=L_{\Omega}\left[L^{-1}\left(s\left(t_{i 1}\right)\right)+L^{-1}\left(s\left(t_{i 2}\right)\right)\right]
$$

Comparing this with the marginal survival from shared frailty approach i.e. $s\left(t_{i 1}\right)=L_{\Omega}\left(\Lambda_{0}\left(t_{i 1}\right)\right)$ and therefore $L_{\Omega}^{-1}\left(s\left(t_{i 1}\right)\right)=\Lambda_{0}\left(t_{i 1}\right)$ shows that

$$
\begin{aligned}
C_{\phi}\left\{s\left(t_{i 1}\right), s\left(t_{i 2}\right)\right\} & =L_{\Omega}\left[L^{-1}\left(s\left(t_{i 1}\right)\right)+L^{-1}\left(s\left(t_{i 2}\right)\right)\right] \\
& =L_{\Omega}\left(\Lambda_{0}\left(t_{i 1}\right)+\Lambda_{0}\left(t_{i 2}\right)\right)=s\left(t_{i 1}, t_{i 2}\right)
\end{aligned}
$$

If $p($.$) is the Laplace transform of a gamma distribution with scale parameter$ 1, then the Clayton copula model is obtained. Similarly, the Gumbel copula is obtained if $p($.$) has a positive stable Laplace though the estimation strategies$ and association measures differ with the frailty approach. Whereas in the copula 
approach, the marginal survivor functions and the dependence structure have to be specified [20] [21] for the joint survivor to be constructed. In frailty models, the dependence structure is introduced indirectly.

\subsection{The Positive Stable Frailty Model}

The probability density function (p.d.f) for the positive stable distribution in closed-form is given by;

$$
f(\Omega)=-\frac{1}{\pi \Omega} \sum_{n=1}^{\infty} \frac{\Gamma(n r+1)}{n !}\left(-\Omega^{-r} k / r\right)^{n} \sin (r n \pi) ; k>0, \Omega>0,0<r \leq 1
$$

For identifiability we assume $k=r$ (see [10] for proof), then we have the standard case with only one parameter $r$.

$$
f(\Omega)=-\frac{1}{\pi \Omega} \sum_{n=1}^{\infty} \frac{\Gamma(n r+1)}{n !}\left(-\Omega^{-r}\right)^{n} \sin (r n \pi) ; \Omega>0,0<r \leq 1
$$

Proposition 2. The Laplace transform is a special case of the Power Variance Family $(r, k, \eta)$ Laplace given by.

$$
L_{\Omega}(s)=\exp \left\{-\frac{k}{r} s^{r}\right\}
$$

As indicated earlier for identifiability reasons we let $k=r$.

$$
L_{\Omega}(s)=\exp \left(-s^{r}\right), 0<r \leq 1
$$

The proposed frailty distribution has many advantages. First, it is easy to implement due to the simplified Laplace transform shown in Equation (11). Second, the positive stable has an infinite mean and variance. This allows for a much higher degree of heterogeneity to be accounted for that would not be possible by using a frailty distribution with finite variance. Third, the positive stable distribution is infinitely divisible, allowing the splitting of the shared risk into cause specific risks which may be easier to interpret. The net bivariate survival, density and hazard functions at time $t_{i 1}>0, t_{i 2}>0$ are:

$$
\begin{gathered}
s\left(t_{i 1}, t_{i 2}\right)=\exp \left\{-\left(\Lambda_{0}\left(t_{i 1}\right)+\Lambda_{0}\left(t_{i 2}\right)\right)^{r}\right\} \\
f\left(t_{i 1}, t_{i 2}\right)=s\left(t_{i 1}, t_{i 2}\right) \cdot \lambda_{0}\left(t_{i 1}\right) \lambda_{0}\left(t_{i 2}\right)\left[r^{2}\left(\Lambda_{0}\left(t_{i 1}\right)+\Lambda_{0}\left(t_{i 2}\right)\right)^{2 r-2}\right. \\
\left.-r(r-1)\left(\Lambda_{0}\left(t_{i 1}\right)+\Lambda_{0}\left(t_{i 2}\right)\right)^{r-2}\right] \\
\lambda\left(t_{i 1}, t_{i 2}\right)=\lambda_{0}\left(t_{i 1}\right) \lambda_{0}\left(t_{i 2}\right)\left[r^{2}\left(\Lambda_{0}\left(t_{i 1}\right)+\Lambda_{0}\left(t_{i 2}\right)\right)^{2 r-2}\right. \\
\left.-r(r-1)\left(\Lambda_{0}\left(t_{i 1}\right)+\Lambda_{0}\left(t_{i 2}\right)\right)^{r-2}\right]
\end{gathered}
$$

In dependence frailty models, the frailty distribution is identifiable through the [9] [22] cross-ratio function, which describes how association of the bivariate hazards evolves over time. The cross ratio measure $A\left(t_{i 1}, t_{i 2}\right)$ for the first life if the second life has experienced the event rather than being event free at a given time is given by; 


$$
A\left(t_{i 1}, t_{i 2}\right)=\frac{s\left(t_{i 1}, t_{i 2}\right) \frac{\partial^{2}}{\partial t_{i 1} \partial t_{i 2}} s\left(t_{i 1}, t_{i 2}\right)}{\frac{\partial}{\partial t_{i 1}} s\left(t_{i 1}, t_{i 2}\right) \frac{\partial}{\partial t_{i 2}} s\left(t_{i 1}, t_{i 2}\right)}
$$

Using the positive stable as frailty distribution, the cross ratio function from Equation (15) becomes:

$$
A\left(t_{i 1}, t_{i 2}\right)=1-\left(1-\frac{1}{r}\right)\left(\Lambda_{0}\left(t_{i 1}\right)+\Lambda_{0}\left(t_{i 2}\right)\right)^{-r}
$$

From Equation (16), values of $r$ close to zero indicate high association between $t_{i 1}$ and $t_{i 2}$ because $A\left(t_{i 1}, t_{i 2}\right)$ takes values greater than $1, r$ close to one indicate low association between $t_{i 1}$ and $t_{i 2}$ since $A\left(t_{i 1}, t_{i 2}\right)$ takes values near 1 while $r=1$ corresponds to independence i.e. $A\left(t_{i 1}, t_{i 2}\right)=1$.

We present below four examples with specific baseline distributions to find the frailty dependence hazard functions with explicit expressions.

\section{Example 1.}

Let $\lambda_{0}(t)$ follow a Weibull $(a, \tau)$ distribution with p.d.f $f_{0}(t)=\tau a t^{\tau-1} \exp \left(-a t^{\tau}\right) ; a, \tau>0 ; t \geq 0$ where $\tau$ is the shape parameter and $a$ the scale parameter.

Then the survival, hazard and cumulative hazard functions are;

1) $s_{0}(t)=\exp \left(-a t^{\tau}\right), t>0$.

2) $\lambda_{0}(t)=\tau a t^{\tau-1}, t>0$.

3) $\Lambda_{0}(t)=a t^{\tau}, t>0$.

From Equation (14) the positive stable Weibull (PSW henceforth) frailty dependence hazard is described explicitly as:

$$
\lambda\left(t_{i 1}, t_{i 2}\right)=a_{1} \tau_{1} t_{i 1}^{\tau_{1}-1} \cdot a_{2} \tau_{2} t_{i 2}^{\tau_{2}-1} \cdot\left[r^{2}\left(a_{1} \tau_{i 1}^{\tau_{1}}+a_{2} t_{i 2}^{\tau_{2}}\right)^{2 r-2}-r(r-1)\left(a_{1} t_{i 1}^{\tau_{1}}+a_{2} t_{i 2}^{\tau_{2}}\right)^{r-2}\right]
$$

The Weibull distribution is widely used in the analysis of lifetime data because it is flexible enough to account for an increasing $(\tau>1)$, decreasing $(\tau<1)$ or constant $(\tau=1)$ hazard rate. Further, the law of Weibull is useful in mortality models for annuitants see e.g. [16].

\section{Example 2.}

Let $\lambda_{0}(t)$ follow a $\operatorname{Lognormal}\left(\mu, \sigma^{2}\right)$ distribution with parameters $\mu, \sigma$ the p.d.f is given by;

$$
f_{0}(t)=\frac{1}{\sigma t \sqrt{2 \pi}} \mathrm{e}^{\frac{-(\ln t-\mu)^{2}}{2 \sigma^{2}}} ; t, \sigma>0,-\infty<\mu<\infty
$$

Then the survival, hazard and cumulative hazard functions are:
1) $s_{0}(t)=1-\Phi\left(\frac{\ln t-\mu}{\sigma}\right), t>0$.
2) $\lambda_{0}(t)=\frac{f_{0}(t)}{1-\Phi\left(\frac{\ln t-\mu}{\sigma}\right)}, t>0$.
3) $\Lambda_{0}(t)=-\ln \left(1-\Phi\left(\frac{\ln t-\mu}{\sigma}\right)\right), t>0$. 
From Equation (14) the positive stable Lognormal frailty dependence hazard is:

$$
\begin{aligned}
\lambda\left(t_{i 1}, t_{i 2}\right)= & \frac{f_{0}\left(t_{i 1}\right)}{1-\Phi\left(\frac{\ln t_{i 1}-\mu_{1}}{\sigma_{1}}\right)} \cdot \frac{f_{0}\left(t_{i 2}\right)}{1-\Phi\left(\frac{\ln t_{i 2}-\mu_{2}}{\sigma_{2}}\right)} \\
& \cdot\left[r^{2}\left(-\ln \left(1-\Phi\left(\frac{\ln t_{i 1}-\mu_{1}}{\sigma_{1}}\right)\right)-\ln \left(1-\Phi\left(\frac{\ln t_{i 2}-\mu_{2}}{\sigma_{2}}\right)\right)\right)^{2 r-2}\right. \\
& \left.-r(r-1)\left(-\ln \left(1-\Phi\left(\frac{\ln t_{i 1}-\mu_{1}}{\sigma_{1}}\right)\right)-\ln \left(1-\Phi\left(\frac{\ln t_{i 2}-\mu_{2}}{\sigma_{2}}\right)\right)\right)^{r-2}\right]
\end{aligned}
$$

The Lognormal is also used in modeling failure time data because it can take various unimodal shapes i.e. bathtub-shaped or hump-shaped.

\section{Example 3.}

Let $\lambda_{0}(t)$ follow a $\operatorname{Gamma}(p, \varphi)$ with p.d.f

$$
f_{0}(t)=\frac{\varphi^{p} t^{p-1} \exp (-\varphi t)}{\Gamma(p)} ; t>0, \varphi>0, p>0
$$

Then the survival, hazard and cumulative hazard functions are:

1) $s_{0}(t)=\frac{\gamma(p, \varphi t)}{\Gamma(p)}, t>0$.

2) $\lambda_{0}(t)=\frac{\varphi^{p} t^{p-1} \exp (-\varphi t)}{\gamma(p, \varphi t)}, t>0$.

3) $\Lambda_{0}(t)=-\ln \left(\frac{\gamma(p, \varphi t)}{\Gamma(p)}\right), t>0$.

The Gamma is widely used in survival analysis to generate mixtures in exponential and Poisson models. It has positive support and is also a good choice for the baseline hazard.

From Equation (14) the positive stable Gamma frailty dependence hazard is described explicitly as:

$$
\begin{aligned}
\lambda\left(t_{i 1}, t_{i 2}\right)= & \frac{\varphi^{p_{1}} t_{i 1}^{p_{1}-1} \exp \left(-\varphi_{1} t_{i 1}\right)}{\gamma\left(p_{1}, \varphi_{1} t_{i 1}\right)} \cdot \frac{\varphi_{2}^{p_{2}} t_{i 2}^{p_{2}-1} \exp \left(-\varphi_{2} t_{i 2}\right)}{\gamma\left(p_{2}, \varphi_{2} t_{i 2}\right)} \\
& \cdot\left[r^{2}\left(-\ln \left(\frac{\gamma\left(p_{1}, \varphi_{1} t_{i 1}\right)}{\Gamma\left(p_{1}\right)}\right)-\ln \left(\frac{\gamma\left(p_{2}, \varphi_{2} t\right)}{\Gamma\left(p_{2}\right)}\right)\right)^{2 r-2}\right. \\
& \left.-r(r-1)\left(-\ln \left(\frac{\gamma\left(p_{1}, \varphi_{1} t_{i 1}\right)}{\Gamma\left(p_{1}\right)}\right)-\ln \left(\frac{\gamma\left(p_{2}, \varphi_{2} t\right)}{\Gamma\left(p_{2}\right)}\right)\right)^{r-2}\right]
\end{aligned}
$$

\subsection{Assessment of Model Selection Criteria}

The performance of the model selection criteria for the Bayesian estimation technique is validated in a comparative study with the traditional MLE method. In the Bayesian method the deviance information criteria (DIC), Bayesian Information Criterion (BIC) and Akaike Information Criterion (AIC) is applied whereas in the MLE method the Standard Error information is used. 
1) $\mathrm{DIC}=\bar{D}+p D$ where: $\bar{D}$ is the posterior mean of $-2 \log L$ measuring the quality of the goodness-of-fit of the considered model to the data.

$\hat{D}=-2 \log L$ is the posterior mean of stochastic nodes and $p D=\bar{D}-\hat{D}$ is the effective number of parameter. Smaller values of DIC indicate better models and could give negative values.

2) $\mathrm{AIC}=\hat{D}+2 p$ where: $p=$ number of parameters of the model.

3) $\mathrm{BIC}=\hat{D}+p \times \log (n)$ where: $p=$ number of parameters of the model and $n=$ sample size. The advantage of the BIC is that it includes the BIC penalty for the number of parameters being estimated.

\section{Bayesian Analysis}

The Bayesian method treats all unknown parameters as random variables in a statistical model and derives their distribution conditional upon known information. This method has been applied in actuarial modeling e.g. by [23] in analysis of simultaneous equations for insurance rate making and by [24] to analyze time-varying dependent data with possible variance shifts. The Bayesian parameter estimation strategy is implemented in the following algorithm run in OpenBUGS: First, the proposal distributions for the likelihood are specified as Weibull $(\tau, a), \operatorname{Lognormal}\left(\mu, \sigma^{2}\right)$ and $\operatorname{Gamma}(p, \varphi)$ respectively. Since we do not have prior information about baseline parameters non-informative prior distribution is picked and assumed to be flat. i.e. gamma distributed random variables with mean 1 and variance 10,000 for positive parameters and normal distribution of mean 1 and variance 10,000 for parameter that can take on positive or negative values. Similar approach is found in [13].

The hyperparameters of initial values are chosen to be MLE estimates determined outside of OpenBUGS using standard techniques e.g. for the Weibull $\tau_{1}=0.7, a_{1}=7$. The actual data to be estimated by the model is specified to be the males and females densities obtained from the AKI 2010 mortality data through standard numerical approximations. Parameters are estimated considering only the range of ages [55, 109]. Burn in period is set at 2000 as per the BGR plot to ensure sequence of draws from the posterior distribution have minimal autocorrelation and can be found by taking values from a single run of the Markov chain. This diminishes the effect of the starting distribution. We run 3 chains in parallel and after 10,000 iterations convergence will be monitored and if stationarity has been achieved (implying estimates are not dependent on the prior distributions) the mean posterior distribution will be picked as a point estimate. Models with smaller values of the DIC, BIC or AIC are preferred.

The WinBUGS codes used to analyze the dataset using Weibull are available upon request.

\section{Brooks-Gelman-Rubin Diagnostic and Trace Plots}

The BGR convergence diagnostic plots for the monitored nodes are presented in Figure 1. As the MCMC simulation progresses, the values of the total-sequence (green curve) and mean within-sequence interval width (blue curve) estimates are monitored. Their ratio (red curve) is seen to converge to one beyond 2000 iterations hence a probable choice for the burn-in period. The dynamic trace 


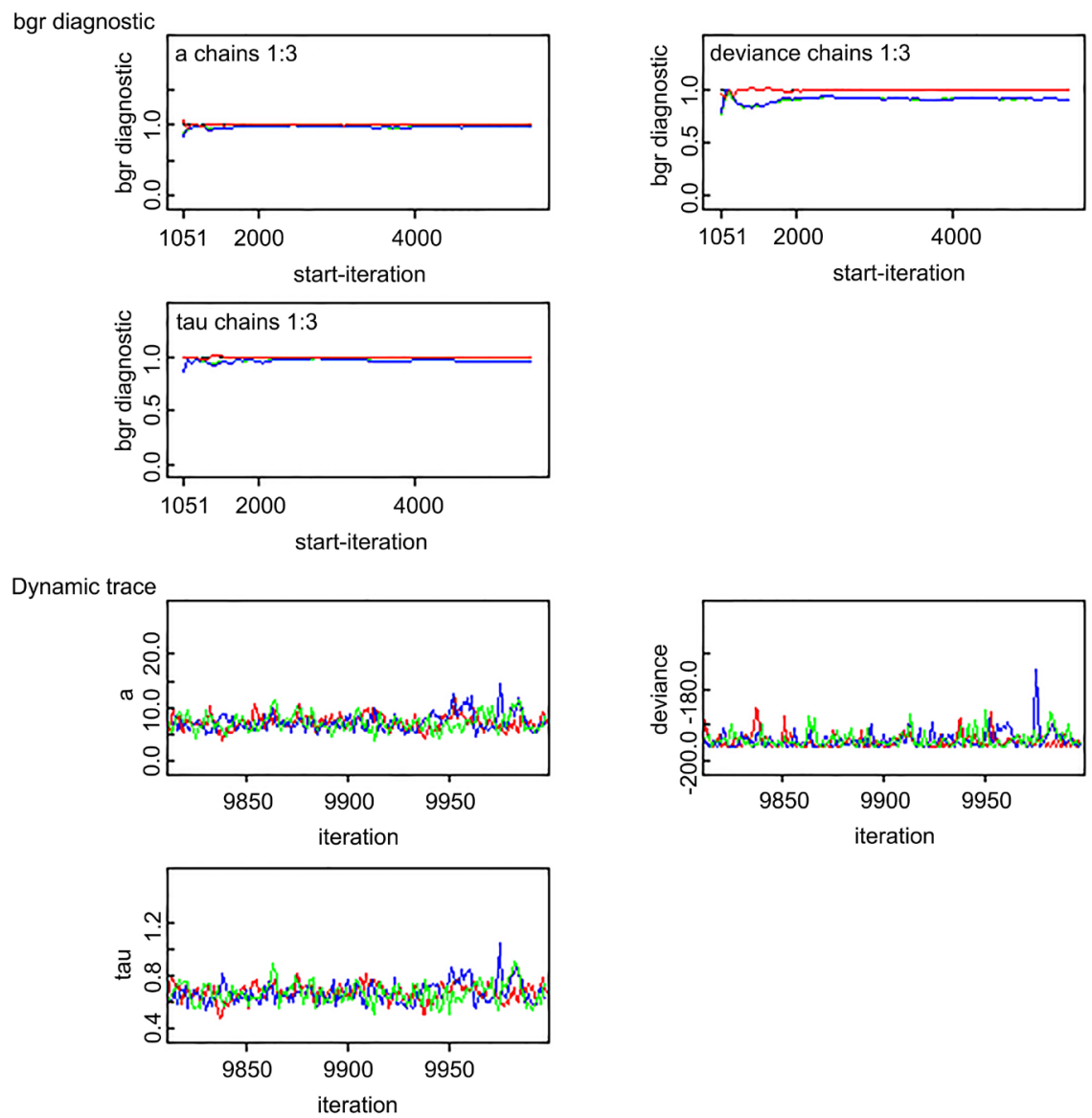

Figure 1. BGR diagnostic plot consistent with convergence and dynamic trace plots.

plots also monitored in Figure 1 is shown to be mean-reverting and the chains appear to mix freely implying stationarity has been achieved.

\section{Comparison with MLE}

The MLE approach is concerned with obtaining parameter values say, $(\tau, a)$ that maximizes the probability of observing the data $D$ given those parameters, $p(D \mid \tau, a)$. The likelihood function gives the probability of the observed sample generated by the model. Generally, maximization of the likelihood function to find the ML estimates is done algebraically, but can be computational intensive. In this article, the MLE algorithm is implemented using MASS package run in $R$. The output is given in Table 2 using the same distribution parametrization as in the Bayesian approach Table 1.

\section{Discussion}

On the basis of Bayesian the Weibull distribution is chosen since the DIC, BIC and AIC are smallest compared to the other distributions. Also using the MLE approach the Weibull is also chosen because the Standard Errors is smallest compared to the other distributions. It is interesting to note from Table 1 and Table 2 that the parameter estimates produced by the two competing approaches agree with minimal deviations. So it may be claimed that Bayesian methods are the potential candidates to be used in any inferential procedures allowing for 
Table 1. Bayesian parameter estimates, DIC, BIC and AIC values.

\begin{tabular}{cccccc}
\hline Baseline Model & \multicolumn{2}{c}{ Parameter Estimates } & DIC & BIC & AIC \\
\hline Weibull & $\tau_{1}=0.67, a_{1}=7.15 \quad$ (male) & -192.2 & -192.82 & -192.2 \\
& $\tau_{2}=0.7555, a_{2}=10.17 \quad$ (female) & -208.9 & -209.52 & -209.0 \\
Lognormal & $\mu_{1}=-3.711, \sigma_{1}^{2}=2.3568$ (male) & -97.22 & -95.75 & -95.24 \\
& $\mu_{2}=-3.727, \sigma_{2}^{2}=1.6697 \quad$ (female) & -101.1 & -99.52 & -99.0 \\
Gamma & $p_{1}=7.746, \varphi_{1}=0.5645$ (male) & -188.6 & -189.22 & -188.7 \\
& $p_{2}=11.89, \varphi_{2}=0.6853$ (female) & -205.5 & -206.02 & -205.5 \\
\hline
\end{tabular}

Table 2. MLE parameter estimates and standard error values.

\begin{tabular}{cccc}
\hline Baseline Model & Parameter Estimates & Standard errors \\
\hline Weibull & $\tau_{1}=0.6720, a_{1}=7.1346 \quad$ (male) & $(0.0687,0.0114)$ \\
Lognormal & $\tau_{2}=0.7558, a_{2}=10.0388 \quad($ female $)$ & $(0.0753,0.0090)$ \\
& $\mu_{1}=-3.7097, \sigma_{1}^{2}=2.31248 \quad$ (male) & $(0.2050,0.1449)$ \\
Gamma & $\mu_{2}=-3.726, \sigma_{2}^{2}=1.63809 \quad$ (female) & $(0.1726,0.1220)$ \\
& $p_{1}=7.830, \varphi_{1}=0.571 \quad$ (male) & $(0.0909,1.8737)$ \\
& $p_{2}=12.0341, \varphi_{2}=0.6935 \quad$ (female) & $(0.1126,2.7592)$ \\
\hline
\end{tabular}

informative priors shared by field experts. The parameter estimates used in the study are as shown in Table 1, following the implementation of Bayesian described.

\section{Goodness of Fit Test}

A chi-square goodness-of-fit test of the data for Weibull baseline distribution is as shown in Table 3. As observed in Table 3, the chi-squared p-value is greater than $5 \%$. Thus, we fail to reject the null hypothesis that the AKI data follow a Weibull distribution at 5\% level of significance. Similarly, the Kolmogorov-Smirnov (KS) hypothesis test in Table 4 between the empirical distribution function and the fitted distribution function shows a p-value greater than 0.05 . We therefore fail to reject the null hypothesis that the AKI data follow a Weibull distribution at 5\% level of significance. The Weibull Q-Q Plot in Figure 2 further shows a straight line through a majority of the quantiles this also shows that the Weibull provides a good fit. We can therefore conclude that the proposed distribution is a good fit for the data.

\section{Model Calibration to the AKI 2010 Male and Female Published Rates}

The PSW dependence model given in Equation (17) is shown in Figure 3 where $\tau_{1}=0.67, a_{1}=7.15 ; \tau_{2}=0.7555, a_{2}=10.17$ obtained from the Bayesian estimation procedure.

Inspired by [3] and for illustration purpose, we discuss the empirical results for the patterns of Equation (17) with different degrees of dependence $r$. We 
Table 3. Goodness of fit of Weibull to the AKI data.

\begin{tabular}{cc}
\hline Name & Value \\
\hline Chi-squared statistic & 2970 \\
Degree of freedom & 2916 \\
Chi-squared p-value & 0.2384 \\
\hline
\end{tabular}

Table 4. Goodness of fit using K-S test.

\begin{tabular}{ccc}
\hline Name & p-value & Test Statistic \\
\hline Kolmogorov-Smirnov test & 0.1463 & 0.21818 \\
\hline
\end{tabular}
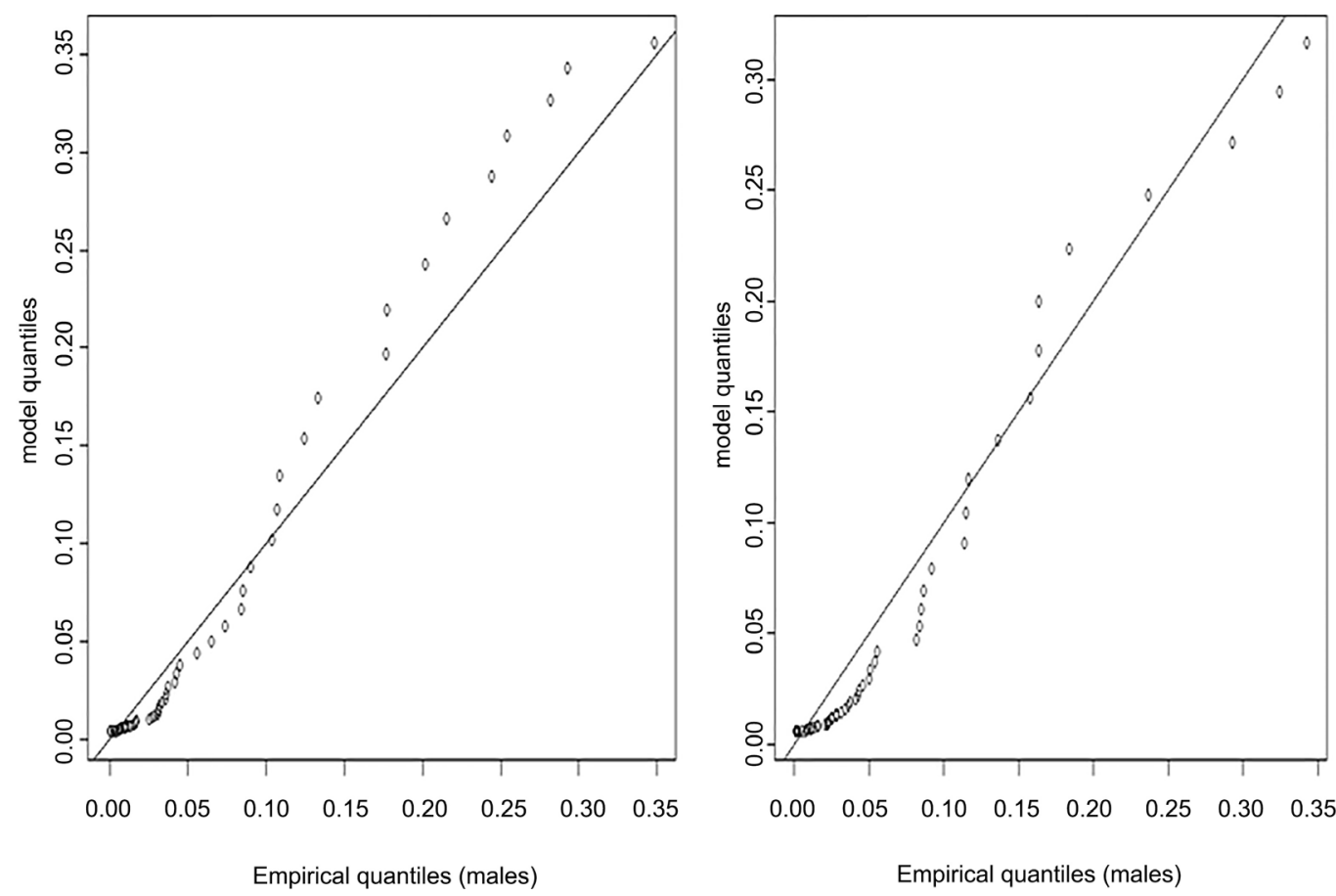

Figure 2. Empirical quantile plot of Weibull to AKI data.

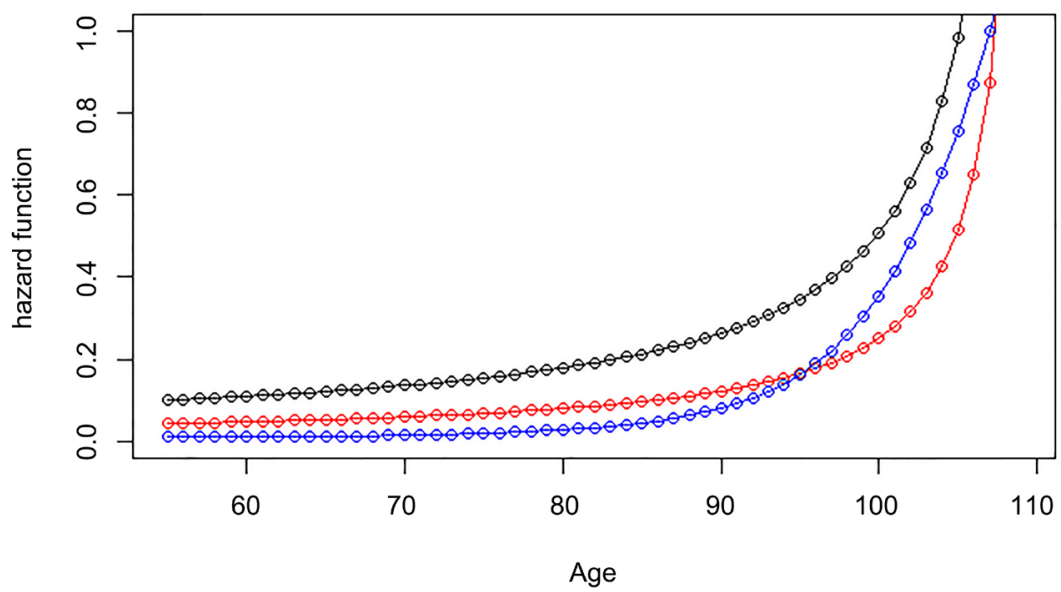

Figure 3. Dependence hazards at various empirical dependence measures. 
consider two dependence measures i.e. Spearman's correlation $\rho=0.74$ (black curve) and Pearson correlation $r=0.68$ (red curve) when the age difference of insured couples is greater than four. As shown in Figure 3, using the above dependence measure shows a significant impact on the hazard compared with the independence (blue curve) assumption. Indeed, one cannot neglect the dependence, even moderate.

Equation (17) is then applied to generate the dependence $\lambda_{x y}$ frailty hazard rates using Pearson correlation $r=0.68$ (to be consistent with the parametric model chosen) shown in Table 5. Real-life data from a Kenyan insurer shows an average age difference of the insured couples to be 6 years. The joint survival probability is $s_{x y}=\exp \left\{-\lambda_{x y}\right.$ frailty $\}$. The EPV for joint-life annuity $a_{x y}$ frailty $=\frac{N_{x y}}{D_{x y}}$ where $D_{x y}=v^{x y} l_{x y}$ and $N_{x y}=\sum_{k=0}^{45} D_{x y+k}$ are commutation functions.

The independence $\lambda_{x y}$ ind hazard rates in Table 5 are obtained by summing the AKI 2010 male and female mortality rates. The survival probability $s_{x y}=s_{x} \cdot s_{y}=\exp \left\{-\lambda_{x y} i n d\right\}$ and EPVs are computed as above. The density function is obtained through standard numerical approximations. Assuming a purchase price of 1000 , the level annuity payments are obtained as indicated.

Table 5. Independence and dependence life-table construction.

\begin{tabular}{|c|c|c|c|c|c|c|c|c|}
\hline \multicolumn{7}{|c|}{ INDEPENDENCE LIFETABLE CONSTRUCTION } & \multicolumn{2}{|c|}{ PURCHASE PRICE: 1000} \\
\hline $\operatorname{AGE}(y)$ & $I_{x y}$ & $S_{x y}$ & $\lambda_{x y}$ ind & $D_{x y}$ & $N_{x y}$ & $a_{x y \_}$ind & PAYMENT STREAM & $\operatorname{AGE}(x)$ \\
\hline 55 & 100,000 & 0.987322 & 0.01276 & $33,650.42$ & $748,844.1$ & 22.25363 & 44.9364899 & 55 \\
\hline 56 & $98,732.15$ & 0.989001 & 0.01106 & $32,572.34$ & $715,193.7$ & 21.95708 & 45.54338661 & 56 \\
\hline 57 & $97,646.23$ & 0.989673 & 0.010381 & $31,582.44$ & $682,621.3$ & 21.61395 & 46.26641343 & 57 \\
\hline 58 & $96,637.84$ & 0.989943 & 0.010108 & $30,643.42$ & $651,038.9$ & 21.24563 & 47.06849595 & 58 \\
\hline 59 & $95,665.97$ & 0.989986 & 0.010064 & $29,740.44$ & $620,395.5$ & 20.86033 & 47.93787231 & 59 \\
\hline 60 & $94,708.01$ & 0.989892 & 0.010159 & $28,865.32$ & 590,655 & 20.46244 & 48.87001675 & 60 \\
\hline 61 & $93,750.7$ & 0.989705 & 0.010348 & $28,013.28$ & $561,789.7$ & 20.0544 & 49.86435956 & 61 \\
\hline 62 & $92,785.53$ & 0.989454 & 0.010602 & $27,181.26$ & $533,776.4$ & 19.63766 & 50.92256147 & 62 \\
\hline 63 & $91,807.06$ & 0.989153 & 0.010906 & $26,367.27$ & $506,595.2$ & 19.21303 & 52.04801895 & 63 \\
\hline 64 & $90,811.23$ & 0.988808 & 0.011255 & $25,569.87$ & $480,227.9$ & 18.781 & 53.24528938 & 64 \\
\hline 65 & $89,794.87$ & 0.988414 & 0.011653 & $24,787.94$ & 454,658 & 18.34191 & 54.51995834 & 65 \\
\hline 66 & $88,754.53$ & 0.987967 & 0.012106 & $24,020.34$ & $429,870.1$ & 17.89608 & 55.87814592 & 66 \\
\hline 67 & $87,686.54$ & 0.98746 & 0.012619 & $23,265.99$ & $405,849.7$ & 17.44391 & 57.32659994 & 67 \\
\hline 68 & $86,586.97$ & 0.986891 & 0.013196 & $22,523.76$ & $382,583.7$ & 16.98579 & 58.87274996 & 68 \\
\hline 69 & $85,451.88$ & 0.986254 & 0.013841 & $21,792.64$ & 360,060 & 16.52209 & 60.52501545 & 69 \\
\hline 70 & $84,277.27$ & 0.985547 & 0.014558 & $21,071.65$ & $338,267.4$ & 16.0532 & 62.29287286 & 70 \\
\hline 71 & $83,059.23$ & 0.984762 & 0.015355 & $20,359.9$ & $317,195.7$ & 15.57943 & 64.18719582 & 71 \\
\hline 72 & $81,793.58$ & 0.983891 & 0.01624 & $19,656.53$ & $296,835.8$ & 15.10113 & 66.22021465 & 72 \\
\hline
\end{tabular}


Continued

\begin{tabular}{|c|c|c|c|c|c|c|c|c|}
\hline 73 & $80,475.98$ & 0.982889 & 0.01726 & $18,960.67$ & $277,179.3$ & 14.61864 & 68.40580518 & 73 \\
\hline 74 & $79,098.91$ & 0.981855 & 0.018312 & $18,270.81$ & $258,218.6$ & 14.13285 & 70.75714002 & 74 \\
\hline 75 & $77,663.65$ & 0.980663 & 0.019526 & $17,587.53$ & $239,947.8$ & 13.64306 & 73.29732669 & 75 \\
\hline 76 & $76,161.88$ & 0.979333 & 0.020884 & $16,909.26$ & $222,360.3$ & 13.15021 & 76.0444313 & 76 \\
\hline 77 & $74,587.82$ & 0.977847 & 0.022402 & $16,235.09$ & 205,451 & 12.65475 & 79.02171141 & 77 \\
\hline 78 & $72,935.47$ & 0.976181 & 0.024108 & $15,564.15$ & $189,215.9$ & 12.15716 & 82.25603251 & 78 \\
\hline 79 & $71,198.19$ & 0.974307 & 0.026029 & $14,895.51$ & $173,651.8$ & 11.65799 & 85.77805174 & 79 \\
\hline 80 & $69,368.91$ & 0.97219 & 0.028204 & $14,228.24$ & $158,756.3$ & 11.15783 & 89.6231621 & 80 \\
\hline 81 & $67,439.76$ & 0.969793 & 0.030673 & $13,561.32$ & 144,528 & 10.65737 & 93.8318065 & 81 \\
\hline 82 & $65,402.58$ & 0.967065 & 0.03349 & $12,893.8$ & $130,966.7$ & 10.15734 & 98.4509591 & 82 \\
\hline 83 & $63,248.52$ & 0.963949 & 0.036716 & $12,224.64$ & $118,072.9$ & 9.658598 & 103.5346902 & 83 \\
\hline 84 & $60,968.37$ & 0.960375 & 0.040431 & $11,552.88$ & $105,848.3$ & 9.162069 & 109.145658 & 84 \\
\hline 85 & $58,552.52$ & 0.95626 & 0.044726 & $10,877.55$ & $94,295.38$ & 8.668808 & 115.3561146 & 85 \\
\hline 86 & $55,991.43$ & 0.951503 & 0.049713 & $10,197.81$ & $83,417.83$ & 8.179977 & 122.2497347 & 86 \\
\hline 87 & $53,276.01$ & 0.945982 & 0.055531 & 9512.983 & $73,220.02$ & 7.696852 & 129.9232519 & 87 \\
\hline 88 & $50,398.16$ & 0.939553 & 0.062351 & 8822.66 & $63,707.04$ & 7.220842 & 138.4879971 & 88 \\
\hline 89 & $47,351.75$ & 0.932044 & 0.070376 & 8126.821 & $54,884.38$ & 6.753487 & 148.0716653 & 89 \\
\hline 90 & $44,133.89$ & 0.923251 & 0.079854 & 7426.03 & $46,757.56$ & 6.29644 & 158.8198986 & 90 \\
\hline 91 & $40,746.67$ & 0.912941 & 0.091084 & 6721.659 & $39,331.53$ & 5.851461 & 170.8975 & 91 \\
\hline 92 & $37,199.3$ & 0.900837 & 0.104431 & 6016.155 & $32,609.87$ & 5.420384 & 184.4888 & 92 \\
\hline 93 & $33,510.49$ & 0.886635 & 0.120322 & 5313.306 & $26,593.71$ & 5.005116 & 199.7956 & 93 \\
\hline 94 & $29,711.59$ & 0.869999 & 0.139263 & 4618.593 & $21,280.41$ & 4.607552 & 217.035 & 94 \\
\hline 95 & $25,849.06$ & 0.85058 & 0.161837 & 3939.385 & $16,661.81$ & 4.229547 & 236.4319 & 95 \\
\hline 96 & $21,986.68$ & 0.827954 & 0.188798 & 3285.059 & $12,722.43$ & 3.872816 & 258.2101 & 96 \\
\hline 97 & $18,203.96$ & 0.802014 & 0.22063 & 2666.546 & 9437.37 & 3.539173 & 282.5519 & 97 \\
\hline 98 & $14,599.82$ & 0.772303 & 0.258379 & 2096.674 & 6770.823 & 3.229317 & 309.663 & 98 \\
\hline 99 & $11,275.48$ & 0.738749 & 0.302797 & 1587.516 & 4674.15 & 2.944316 & 339.6374 & 99 \\
\hline 100 & 8329.751 & 0.701364 & 0.354728 & 1149.78 & 3086.634 & 2.684542 & 372.503 & 100 \\
\hline 101 & 5842.187 & 0.660341 & 0.414999 & 790.6024 & 1936.854 & 2.449845 & 408.189 & 101 \\
\hline 102 & 3857.834 & 0.616061 & 0.48441 & 511.8304 & 1146.251 & 2.239514 & 446.5255 & 102 \\
\hline 103 & 2376.66 & 0.569077 & 0.56374 & 309.1358 & 634.4207 & 2.05224 & 487.2725 & 103 \\
\hline 104 & 1352.502 & 0.519526 & 0.654838 & 172.4726 & 325.2849 & 1.88601 & 530.2199 & 104 \\
\hline 105 & 702.6603 & 0.469801 & 0.755445 & 87.84709 & 152.8124 & 1.739527 & 574.869 & 105 \\
\hline 106 & 330.1108 & 0.419039 & 0.869791 & 40.46146 & 64.96529 & 1.605609 & 622.8165 & 106 \\
\hline 107 & 138.3293 & 0.368476 & 0.99838 & 16.62247 & 24.50383 & 1.474139 & 678.3623 & 107 \\
\hline 108 & 50.97098 & 0.318742 & 1.143375 & 6.004881 & 7.881357 & 1.312492 & 761.9096 & 108 \\
\hline 109 & 16.24657 & 0.27038 & 1.307927 & 1.876476 & 1.876476 & 1 & 1000 & 109 \\
\hline
\end{tabular}




\section{Continued}

\begin{tabular}{|c|c|c|c|c|c|c|c|c|}
\hline \multicolumn{6}{|c|}{ INDEPENDENCE LIFETABLE CONSTRUCTION } & \multicolumn{3}{|c|}{ PURCHASE PRICE: 1000} \\
\hline $\operatorname{AGE}(y)$ & $I_{x y}$ & $S_{x y}$ & $\mu_{x y}$ frailty & $D_{x y}$ & $N_{x y}$ & $a_{x y}$ frailty & PAYMENT STREAM & $\operatorname{AGE}(x)$ \\
\hline 55 & 100,000 & 0.956844 & 0.044115 & $33,650.42$ & $447,990.3$ & 13.31306 & 75.11419 & 55 \\
\hline 56 & $95,684.42$ & 0.956038 & 0.044957 & $31,566.88$ & $414,339.8$ & 13.12578 & 76.18596 & 56 \\
\hline 57 & $91,477.96$ & 0.955202 & 0.045832 & $29,587.39$ & 382,773 & 12.93703 & 77.2975 & 57 \\
\hline 58 & $87,379.94$ & 0.954334 & 0.046742 & $27,707.78$ & $353,185.6$ & 12.7468 & 78.45107 & 58 \\
\hline 59 & $83,389.65$ & 0.953432 & 0.047687 & 25,924 & $325,477.8$ & 12.55507 & 79.64907 & 59 \\
\hline 60 & $79,506.39$ & 0.952495 & 0.048671 & $24,232.14$ & $299,553.8$ & 12.36184 & 80.89411 & 60 \\
\hline 61 & $75,729.42$ & 0.951519 & 0.049695 & $22,628.42$ & $275,321.6$ & 12.16708 & 82.18902 & 61 \\
\hline 62 & $72,058.01$ & 0.950504 & 0.050763 & $21,109.19$ & $252,693.2$ & 11.97077 & 83.53683 & 62 \\
\hline 63 & $68,491.4$ & 0.949445 & 0.051877 & $19,670.95$ & 231,584 & 11.7729 & 84.94086 & 63 \\
\hline 64 & $65,028.85$ & 0.948341 & 0.053041 & $18,310.28$ & $211,913.1$ & 11.57345 & 86.40468 & 64 \\
\hline 65 & $61,669.55$ & 0.947189 & 0.054256 & $17,023.92$ & $193,602.8$ & 11.3724 & 87.93221 & 65 \\
\hline 66 & $58,412.73$ & 0.945985 & 0.055528 & $15,808.7$ & $176,578.9$ & 11.16973 & 89.5277 & 66 \\
\hline 67 & $55,257.57$ & 0.944726 & 0.056861 & $14,661.56$ & $160,770.2$ & 10.96542 & 91.1958 & 67 \\
\hline 68 & $52,203.26$ & 0.943407 & 0.058257 & $13,579.57$ & $146,108.6$ & 10.75945 & 92.94159 & 68 \\
\hline 69 & $49,248.94$ & 0.942026 & 0.059723 & $12,559.87$ & 132,529 & 10.55179 & 94.77068 & 69 \\
\hline 70 & $46,393.77$ & 0.940576 & 0.061263 & $11,599.72$ & $119,969.2$ & 10.34242 & 96.6892 & 70 \\
\hline 71 & $43,636.86$ & 0.939053 & 0.062883 & $10,696.49$ & $108,369.5$ & 10.13131 & 98.70393 & 71 \\
\hline 72 & $40,977.33$ & 0.937451 & 0.06459 & 9847.62 & $97,672.96$ & 9.918434 & 100.8224 & 72 \\
\hline 73 & $38,414.25$ & 0.935765 & 0.066391 & 9050.652 & $87,825.34$ & 9.703759 & 103.0528 & 73 \\
\hline 74 & $35,946.71$ & 0.933986 & 0.068294 & 8303.217 & $78,774.69$ & 9.48725 & 105.4046 & 74 \\
\hline 75 & $33,573.73$ & 0.932108 & 0.070306 & 7603.03 & $70,471.47$ & 9.268867 & 107.8881 & 75 \\
\hline 76 & $31,294.35$ & 0.930122 & 0.07244 & 6947.889 & $62,868.44$ & 9.048568 & 110.5147 & 76 \\
\hline 77 & $29,107.56$ & 0.928018 & 0.074704 & 6335.671 & $55,920.56$ & 8.826304 & 113.2977 & 77 \\
\hline 78 & $27,012.35$ & 0.925786 & 0.077112 & 5764.331 & $49,584.89$ & 8.602019 & 116.2518 & 78 \\
\hline 79 & $25,007.65$ & 0.923413 & 0.079678 & 5231.898 & $43,820.55$ & 8.375651 & 119.3937 & 79 \\
\hline 80 & $23,092.4$ & 0.920887 & 0.082418 & 4736.475 & $38,588.66$ & 8.147125 & 122.7427 & 30 \\
\hline 81 & $21,265.48$ & 0.918191 & 0.08535 & 4276.232 & $33,852.18$ & 7.916357 & 126.3207 & 81 \\
\hline 82 & $19,525.77$ & 0.915308 & 0.088495 & 3849.409 & $29,575.95$ & 7.683245 & 130.1533 & 82 \\
\hline 83 & $17,872.09$ & 0.912218 & 0.091876 & 3454.308 & $25,726.54$ & 7.447669 & 134.2702 & 83 \\
\hline 84 & $16,303.24$ & 0.908899 & 0.095522 & 3089.296 & $22,272.23$ & 7.209484 & 138.7062 & 84 \\
\hline 85 & $14,817.99$ & 0.905323 & 0.099464 & 2752.801 & $19,182.94$ & 6.968515 & 143.5026 & 85 \\
\hline 86 & $13,415.07$ & 0.90146 & 0.103739 & 2443.308 & $16,430.14$ & 6.724546 & 148.7089 & 36 \\
\hline 87 & $12,093.15$ & 0.897275 & 0.108393 & 2159.358 & $13,986.83$ & 6.477309 & 154.3851 & 87 \\
\hline 88 & $10,850.89$ & 0.892726 & 0.113476 & 1899.547 & $11,827.47$ & 6.226468 & 160.6047 & 88 \\
\hline 89 & 9686.864 & 0.887762 & 0.119051 & 1662.524 & 9927.922 & 5.971597 & 167.4594 & 89 \\
\hline 90 & 8599.633 & 0.882327 & 0.125193 & 1446.986 & 8265.398 & 5.712147 & 175.0655 & 90 \\
\hline
\end{tabular}


Continued

\begin{tabular}{|c|c|c|c|c|c|c|c|c|}
\hline 91 & 7587.685 & 0.876348 & 0.131992 & 1251.681 & 6818.412 & 5.447404 & 183.5737 & 91 \\
\hline 92 & 6649.455 & 0.869743 & 0.139558 & 1075.401 & 5566.731 & 5.176426 & 193.1835 & 92 \\
\hline 93 & 5783.314 & 0.862406 & 0.148029 & 916.982 & 4491.33 & 4.897948 & 204.1671 & 93 \\
\hline 94 & 4987.565 & 0.845001 & 0.157576 & 775.3048 & 3574.348 & 4.61025 & 216.908 & 94 \\
\hline 95 & 4214.5 & 0.834576 & 0.168417 & 642.2878 & 2799.044 & 4.357927 & 229.4669 & 95 \\
\hline 96 & 3517.318 & 0.82268 & 0.180832 & 525.5272 & 2156.756 & 4.103985 & 243.6656 & 96 \\
\hline 97 & 2893.626 & 0.808983 & 0.195189 & 423.8632 & 1631.229 & 3.848479 & 259.8429 & 97 \\
\hline 98 & 2340.894 & 0.793049 & 0.211977 & 336.1746 & 1207.366 & 3.591484 & 278.4365 & 98 \\
\hline 99 & 1856.444 & 0.774291 & 0.23187 & 261.3755 & 871.1909 & 3.3331 & 300.021 & 99 \\
\hline 100 & 1437.429 & 0.751898 & 0.255807 & 198.4125 & 609.8154 & 3.073472 & 325.3649 & 100 \\
\hline 101 & 1080.8 & 0.724725 & 0.285154 & 146.2608 & 411.4029 & 2.812803 & 355.5173 & 101 \\
\hline 102 & 783.2826 & 0.6911 & 0.321963 & 103.9204 & 265.142 & 2.551394 & 391.9425 & 102 \\
\hline 103 & 541.3263 & 0.648499 & 0.369471 & 70.41114 & 161.2216 & 2.289717 & 436.7352 & 103 \\
\hline 104 & 351.0496 & 0.592961 & 0.433095 & 44.76624 & 90.81045 & 2.028548 & 492.9635 & 104 \\
\hline 105 & 208.1588 & 0.518012 & 0.522626 & 26.02416 & 46.04421 & 1.769287 & 565.1995 & 105 \\
\hline 106 & 107.8287 & 0.41282 & 0.657758 & 13.21649 & 20.02004 & 1.514778 & 660.1627 & 106 \\
\hline 107 & 44.5138 & 0.260909 & 0.884744 & 5.349046 & 6.803557 & 1.27192 & 786.2131 & 107 \\
\hline 108 & 11.61405 & 0.064307 & 1.343584 & 1.368249 & 1.454511 & 1.063046 & 940.6933 & 108 \\
\hline 109 & 0.746861 & 1 & 2.744092 & 0.086262 & 0.086262 & 1 & 1000 & 109 \\
\hline
\end{tabular}

\section{Impact on Mortality Rates}

As shown in Table $5 \lambda_{x y}$ ind is lower than $\lambda_{x y}$ frailty during the early annuitants ages. This can be attributed to negative effects of dependence accounted for in the frailty model, thereby accounting for lower-tail dependence that is present. Thereafter, there is an overestimation of mortality risk in the independence model compared to the dependence model due to longevity risk (positive effects of dependence). Here, the upper-tail dependence has been accounted for. Thus the independence assumption underestimates deceleration in the mortality increase at very old ages.

\section{Impact on Annuity EPVs}

Consequently, comparing the annuity EPVs and payment streams shows that the independence assumptions lead to an overestimation of the insurer's liability at the initial stages of the contract thereafter there is an underestimation of liability due to deceleration in the mortality increase at very old ages (longevity risk).

\section{Concluding Remarks}

Although there is rich literature in frailty dependence modeling, most applications have been in medical field. Various dependence models have been considered in actuarial literature; however, the focus has been on either the lower-tail 
dependence or upper-tail dependence.

This article presents the frailty dependence approach calibrated on the AKI 2010 male and female published mortality rates due to limited joint-life mortality data-set available in the Kenyan market. This methodology offers greater flexibility than the lower-tail or upper-tail dependence models while preserving closed-form expressions for the net survival functions. Our strategy is to apply the conditional independence assumption in a positive stable frailty approach to account for lower and upper-tail dependence. A positive stable frailty approach is then applied to construct dependence life-tables. The frailty joint-life mortality rates are proposed to generate life annuity payment streams in the competitive Kenyan market.

The conclusion reached is that comparing the independence mortality assumption with the dependence frailty model shows a decrease in the insurer's expected liability at the early annuitant's ages followed by an increase at later ages when dependence is accounted for. This can be explained by the fact that the frail couples shall have died during the early stages of the contract, thereafter deceleration in the mortality increase at very old ages (longevity risk), underscoring the importance of dependence modeling in pricing insurance products. Thus, assuming the joint lives to be independent could lead to biased annuity valuations.

\section{Acknowledgements}

We would like to acknowledge the Association of Kenya Insurer for the study conducted on assured lives during 2010 whose mortality rates are currently being used by insurance companies in Kenya and have been used in the study.

\section{Authors' Contribution}

All authors have contributed equally in the development of this article.

\section{Conflicts of Interest}

The authors declare no conflicts of interest regarding the publication of this paper.

\section{References}

[1] Olivieri, A. and Pitacco, E. (1999) Funding Sickness Benefits for the Elderly. Proceedings of the 30 th ASTIN Colloquium, Tokyo, 22-25 August 1999, 135-155.

[2] Coppola, M., Di Lorenzo, E. and Sibillo, M. (2000) Risk Sources in a Life Annuity Portfolio: Decomposition and Measurement Tools. Journal of Actuarial Practice, 8, 43-61.

[3] Gildas, R., Francois, D., Enkelejd, H. and Youssouf, T. (2018) On Age Difference in Joint Lifetime Modeling with Life Assurance Annuity Applications. Annals of Actuarial Science, 12, 350-371. https://doi.org/10.1017/S1748499518000076

[4] Meyricke, R. and Sherris, M. (2013) The Determinants of Mortality Heterogeneity and Implications for Pricing Annuities. Insurance: Mathematics and Economics, 53, 
379-387. https://doi.org/10.1016/j.insmatheco.2013.06.002

[5] Olivieri, A. and Pitacco, E. (2016) Frailty and Risk Classification for Life Annuity Portfolios. Risks, 4, 39. https://doi.org/10.3390/risks4040039

[6] Luciano, E., Vigna, E. and Spreeuw, J.S. (2016) Dependence across Generations and Pricing Impact on Reversionary Annuities. Risks, 4, 16. https://doi.org/10.3390/risks4020016

[7] Yang, L. (2017) Broken-Heart, Common Life, Heterogeneity: Analyzing the Spousal Mortality Dependence. ASTIN Bulletin: The Journal of the IAA, 47, 837-874. https://doi.org/10.1017/asb.2017.8

[8] D'Amato, V., Haberman, S. and Piscopo, G. (2017) The Dependency Premium Based on a Multifactor Model for Dependent Mortality Data. Communications in Statistics-Theory and Methods, 48, 50-61. https://doi.org/10.1080/03610926.2017.1366523

[9] Clayton, D.G. (1978) A Model for Association in Bivariate Lifetables and Its Application in Epidemiological Studies of Familial Tendency in Chronic Disease Incidence. Biometrika, 65, 141-151. https://doi.org/10.1093/biomet/65.1.141

[10] Hougaard, P. (2000) Analysis of Multivariate Survival Data. Springer, New York. https://doi.org/10.1007/978-1-4612-1304-8

[11] Fulla, S. and Laurent, P. (2008) Mortality Fluctuations Modelling with a Shared Frailty Approach.

[12] Wienke, A. (2010) Frailty Models in Survival Analysis. CRC Press, New York. https://doi.org/10.1201/9781420073911

[13] Hanagal, D. (2020) Correlated Positive Stable Frailty Models. Communications in Statistics-Theory and Methods, 47, 1-17. https://doi.org/10.1080/03610926.2020.1736305

[14] Brouhns, N., Denuit, M. and Vermunt, K.J. (2002) A Poisson Log-Bilinear Regression Approach to the Construction of Projected Lifetables. Insurance: Mathematics and Economics, 31, 373-393. https://doi.org/10.1016/S0167-6687(02)00185-3

[15] Wang, S.S. and Brown, R.L. (1998) A Frailty Model for Projection of Human Mortality Improvements. Journal of Actuarial Practice, 6, 221-241. http://digitalcommons.unl.edu/joap/95

[16] Olivieri, A. (2001) Uncertainty in Mortality Projections: An Actuarial Perspective. Insurance: Mathematics and Economics, 29, 231-245. https://doi.org/10.1016/S0167-6687(01)00084-1

[17] Carriere, J.F. (2000) Bivariate Survival Models for Coupled Lives. Scandinavian Actuarial Journal, 1, 17-31. https://doi.org/10.1080/034612300750066700

[18] Cossette, H., Marceau, E., Mtalai, I. and Veilleux, D. (2017) Dependent Risk Models with Archimedean Copulas: A Computational Strategy Based on Common Mixtures and Applications. Insurance: Mathematics and Economics, 78, 53-71. https://doi.org/10.1016/j.insmatheco.2017.11.002

[19] Hong, L. and Yang, L. (2018) Modeling Cause-of-Death Mortality Using Hierarchical Archimedean Copula. Scandinavian Actuarial Journal, No. 3, 247-272. https://doi.org/10.1080/03461238.2018.1546224

[20] Nelsen, R.B. (2007) An Introduction to Copulas. Springer Science and Business Media, Berlin.

[21] Czado, C., Kastenmeier, R., Brechmann, E.C. and Min, A. (2012) A Mixed Copula Model for Insurance Claims and Claim Sizes. Scandinavian Actuarial Journal, 4, 278-305. https://doi.org/10.1080/03461238.2010.546147 
[22] Oakes, D. (1989) Bivariate Survival Models Induced by Frailties. Journal of the American Statistical Association, 84, 487-493.

https://doi.org/10.1080/01621459.1989.10478795

[23] Scollnik, D.P.M. (1993) A Bayesian Analysis of a Simultaneous Equations Model for Insurance Rate-Making. Insurance: Mathematics and Economics, 12, 265-286. https://doi.org/10.1016/0167-6687(93)90238-K

[24] Rosenberg, M. and Young, V.R. (1999) A Bayesian Approach to Understanding Time Series Data. North American Actuarial Journal, 3, 130-143.

https://doi.org/10.1080/10920277.1999.10595808 